PROCEEDINGS OF THE

AMERICAN MATHEMATICAL SOCIETY

Volume 134, Number 1, Pages 1-3

S 0002-9939(05)08289-4

Article electronically published on August 19, 2005

\title{
MULTIPLICITIES OF REPRESENTATIONS IN SPACES OF MODULAR FORMS
}

\author{
BIN YONG HSIE
}

(Communicated by David E. Rohrlich)

\begin{abstract}
This paper shows that for a given irreducible representation $\rho$ of $\Gamma / \Gamma_{1}$, the two functions $\operatorname{dim}\left(M_{k}\left(\Gamma_{1}, \rho\right)\right)$ and $\operatorname{dim}\left(S_{k}\left(\Gamma_{1}, \rho\right)\right)$ of $k$ are almost linear functions.
\end{abstract}

Let $\Gamma$ and $\Gamma_{1}$ be two Fuchsian subgroups of $\mathrm{SL}_{2}(\mathbb{Q})$ of the first kind, with $\Gamma_{1}$ a normal subgroup of $\Gamma$ of index $\mu$. Let $\mathbb{H}^{*}$ denote the extended upper half-plane as in Shimura [4] and put $Y=\Gamma \backslash \mathbb{H}^{*}$ and $X=\Gamma_{1} \backslash \mathbb{H}^{*}$. Let $\Omega(X)$ and $\Omega(Y)$ be the fields of meromorphic functions of $X$ and $Y$ respectively. Then $A_{0}(\Gamma) \cong \Omega(Y)$ and $A_{0}\left(\Gamma_{1}\right) \cong \Omega(X)$ in a natural way, where $A_{k}(*)$ denotes the space of meromorphic modular forms for $*$ of weight $k$. We define a representation $\pi_{k}$ of $\Gamma$ on $A_{k}\left(\Gamma_{1}\right)$ by the formula

$$
\pi_{k}(\gamma)(f)=f \mid\left[\gamma^{-1}\right]_{k} \quad\left(\gamma \in \Gamma, \quad f \in A_{k}\left(\Gamma_{1}\right)\right),
$$

where the notation $[*]_{k}$ is as in [4]. The representation $\pi_{k}$ factors through $\Gamma / \Gamma_{1}$ to give a representation - also denoted $\pi_{k}$-of the latter group. The space $M_{k}\left(\Gamma_{1}\right)$ of holomorphic modular forms of weight $k$ for $\Gamma_{1}$ and the subspace $S_{k}\left(\Gamma_{1}\right)$ of cusp forms of weight $k$ for $\Gamma_{1}$ are both stable under $\pi_{k}$, and the resulting representations of $\Gamma / \Gamma_{1}$ on $M_{k}\left(\Gamma_{1}\right)$ and $S_{k}\left(\Gamma_{1}\right)$ will be denoted $\rho_{k}$ and $\sigma_{k}$ respectively.

Henceforth, $\rho$ denotes an irreducible complex representation of $\Gamma / \Gamma_{1}$. If $-I \in \Gamma$, then the value of $\rho$ on the coset of $-I$ in $\Gamma / \Gamma_{1}$ is a scalar by Schur's lemma, and we say that $\rho$ is even or odd according to whether the scalar is 1 or -1 . If $-I \in \Gamma_{1}$, then $\rho$ is automatically even.

If $\pi$ is any finite-dimensional complex representation of $\Gamma / \Gamma_{1}$, then we write $\langle\rho, \pi\rangle$ for the multiplicity of $\rho$ in $\pi$. For example, $\left\langle\rho, \rho_{\text {reg }}\right\rangle=\operatorname{dim} \rho$, where $\rho_{\text {reg }}$ is the regular representation of $\Gamma / \Gamma_{1}$.

Theorem. Fix an irreducible complex representation $\rho$ of $\Gamma / \Gamma_{1}$, and put

$$
c=\frac{1}{4 \pi} \int_{\Gamma \backslash \mathbb{H}} \frac{d x d y}{y^{2}} .
$$

If $-I \notin \Gamma$, then

$$
\lim _{k \rightarrow \infty} \frac{\left\langle\rho, \rho_{k}\right\rangle}{k\left\langle\rho, \rho_{\text {reg }}\right\rangle}=\lim _{k \rightarrow \infty} \frac{\left\langle\rho, \sigma_{k}\right\rangle}{k\left\langle\rho, \rho_{\text {reg }}\right\rangle}=c .
$$

If $-I \in \Gamma$, then the same assertion holds provided $k$ runs through positive integers of the same parity as $\rho$.

Received by the editors June 4, 2004.

2000 Mathematics Subject Classification. Primary 11F11.

Key words and phrases. Modular form, cusp form.

(C)2005 American Mathematical Society 
Proof. We shall prove our assertion only for $\rho_{k}$. The proof for $\sigma_{k}$ is similar.

For any positive integer $k$, let $i(k)=(-1)^{k}$. If $-I \in \Gamma_{1}$, we assume $k$ is always even.

Let $\mathcal{S}$ be the set of the irreducible representations of $\Gamma / \Gamma_{1}$ with the same parity as $k$ if $-I \in \Gamma$, and the set of all irreducible representations of $\Gamma / \Gamma_{1}$ otherwise.

Let $p$ be a non-cusp point of $Y$ which has $\mu$ different liftings $p_{1}, \ldots, p_{\mu}$ on $X$, and let $\tilde{p}_{j}$ be a lifting of $p_{j}$ in $\mathbb{H}^{*}(1 \leq j \leq \mu)$. By the Riemann-Roch theorem we know that there exists an $f \in A_{i(k)}\left(\Gamma_{1}\right)$ such that $f$ has poles only at cusps and $f\left(\tilde{p}_{1}\right)=1, f\left(\tilde{p}_{j}\right)=0$ for all $j=2, \ldots, \mu$. For any $\alpha \in \Gamma / \Gamma_{1}$, put $f_{\alpha}=f \mid[\alpha]_{i(k)}$. For a fixed sufficiently large even $n_{0}$, we may choose $\varphi=\varphi_{n_{0}} \in S_{n_{0}}(\Gamma)$ such that $\varphi f_{\alpha}\left(\alpha \in \Gamma / \Gamma_{1}\right)$ are all cusp forms. Let $W$ denote the $\mathbb{C}$-linear subspace of $M_{i(k)+n_{0}}\left(\Gamma_{1}\right)$ spanned by $\left\{\varphi f_{\alpha} \mid \alpha \in \Gamma / \Gamma_{1}\right\}$. Clearly, $W$ is stable under $\pi_{i(k)+n_{0}}$. It is easy to see that

$$
W \cong \bigoplus_{\rho \in \mathcal{S}}\left\langle\rho, \rho_{\mathrm{reg}}\right\rangle \rho
$$

since the two-hand sides have the same trace.

When $k \geq i(k)+n_{0}$, the choice of $f$ ensures that the natural map

$$
W \otimes_{\mathbb{C}} M_{k-i(k)-n_{0}}(\Gamma) \rightarrow M_{k}\left(\Gamma_{1}\right)
$$

is injective. Hence,

$$
\frac{\left\langle\rho, \rho_{k}\right\rangle}{k\left\langle\rho, \rho_{\text {reg }}\right\rangle} \geq \frac{\operatorname{dim}\left(M_{k-i(k)-n_{0}}(\Gamma)\right)}{k} .
$$

For $\rho$ and $k$ as in the theorem, in [4] Shimura showed that

$$
\lim _{k \rightarrow \infty} \frac{\operatorname{dim}\left(M_{k-i}(\Gamma)\right)}{k}=c
$$

and

$$
\lim _{k \rightarrow \infty} \frac{\operatorname{dim}\left(M_{k}\left(\Gamma_{1}\right)\right)}{k}=c \mu .
$$

From (1) and (2) we easily deduce that

$$
\liminf _{k \rightarrow \infty} \frac{\left\langle\rho, \rho_{k}\right\rangle}{k} \geq c\left\langle\rho, \rho_{\text {reg }}\right\rangle .
$$

It is obvious that (3) is equivalent to

$$
\lim _{k \rightarrow \infty} \sum_{\rho \in \mathcal{S}} \operatorname{dim}(\rho) \frac{\left\langle\rho, \rho_{k}\right\rangle}{k}=\sum_{\rho \in \mathcal{S}} \operatorname{dim}(\rho) c\left\langle\rho, \rho_{\mathrm{reg}}\right\rangle .
$$

Combining this equality with (4), we get

$$
\lim _{k \rightarrow \infty} \frac{\left\langle\rho, \rho_{k}\right\rangle}{k}=c\left\langle\rho, \rho_{\text {reg }}\right\rangle,
$$

as desired.

\section{ACKNOWLEDGEMENT}

I would like to thank Professor David E. Rohrlich for his kind help in revising this paper. 


\section{REFERENCES}

1. O. Forster, Lectures on Riemann Surfaces, Springer-Verlag, New York, 1981. MR0648106 (83d:30046)

2. N. Koblitz, Introduction to Elliptic Curves and Modular Forms, Springer-Verlag, New York, 1984. MR.0766911 (86c:11040)

3. J-P. Serre, Linear Representations of Finite Groups, Springer-Verlag, New York, 1977. MR:0450380 (56:8675)

4. G. Shimura, Introduction to the Arithmetic Theory of Automorphic Functions, Princeton University Press, Princeton, New Jersey, 1971.

Lmam, Department of Mathematics, Peking University, Beijing 100871, People's RePUBLIC OF CHINA

E-mail address: byhsie@math.pku.edu.cn 